\title{
Growth and Laboratory Maintenance of Campylobacter jejuni
}

\author{
Lindsay Davis ${ }^{1}$ and Victor DiRita ${ }^{1,2}$ \\ ${ }^{1}$ Department of Microbiology and Immunology, University of Michigan, Ann Arbor, \\ Michigan \\ ${ }^{2}$ Unit for Laboratory Animal Medicine, University of Michigan, Ann Arbor, Michigan
}

\begin{abstract}
Campylobacter jejuni is a fastidious organism, growing in microaerophilic conditions with a temperature range between $37^{\circ}$ and $42^{\circ} \mathrm{C}$. Multiple types of media can be used to cultivate it; however, Mueller Hinton broth and agar support the best $C$. jejuni growth. Optimum atmosphere for C. jejuni is $85 \% \mathrm{~N}_{2}, 10 \% \mathrm{CO}_{2}$, and $5 \% \mathrm{O}_{2}$. Curr. Protoc. Microbiol. 10:8A.1.1-8A.1.7. (C) 2008 by John Wiley \& Sons, Inc.
\end{abstract}

Keywords: campylobacteriosis • microaerophilic growth $\bullet$ Mueller Hinton

\section{INTRODUCTION}

Campylobacter jejuni is one of the major causes of bacterial gastroenteritis worldwide and is primarily acquired through the ingestion of contaminated poultry products. Research on $C$. jejuni has been greatly impaired due to poor culturing techniques and genetic tools. C. jejuni was successfully isolated in the early 1970s, utilizing specialty agar and microaerophilic atmospheric conditions. Since its isolation, it has been connected with bacterial gastroenteritis as well as the neurological disorders Guillen-Barré Syndrome and Fisher Syndrome in humans. It is now one of the leading causes of gastroenteritis in both the developed and developing worlds. (Young et al., 2007).

C. jejuni is a fastidious organism, requiring modified atmospheric conditions, a longer growing time, a narrow temperature range, and specialized media when compared to such bacteria as E. coli. All these factors must be taken into consideration when working with $C$. jejuni.

\section{STRATEGIC PLANNING}

\section{Atmosphere}

Campylobacter jejuni requires microaerophilic conditions for growth. Optimum growth is maintained in a tri-gas incubator (e.g., Thermo Forma Series II Water-Jacketed $\mathrm{CO}_{2}$ Incubator), with $85 \% \mathrm{~N}_{2}, 10 \% \mathrm{CO}_{2}$, and $5 \% \mathrm{O}_{2}$. If a tri-gas incubator is unavailable, specific gas packs (i.e., BBL Campy Pak Plus) or formulated compressed air can be applied with sealable gas chambers or plastic bags, respectively. Gas packs are expensive, and growth in plastic bags is suboptimal compared to that obtained by using a tri-gas incubator. Notes concerning the differences are in the specific protocols outlined. $C$. jejuni is also able to grow in anaerobic conditions.

\section{Temperature}

C. jejuni has a narrow temperature range of growth, with optimum growth occurring between $37^{\circ}$ and $42^{\circ} \mathrm{C}$. The bacteria are able to survive at $7^{\circ} \mathrm{C}$ and perform vital cellular processes such as protein synthesis; however, growth and recovery at low temperatures

Current Protocols in Microbiology 8A.1.1-8A.1.7, August 2008

Published online August 2008 in Wiley Interscience (www.interscience.wiley.com).

DOI: $10.1002 / 9780471729259 . m c 08 a 01 s 10$

Copyright (C) 2008 John Wiley \& Sons, Inc.
UNIT 8A.1

Epsilon

Proteobacteria

8A.1.1

Supplement 10 
is difficult (Hazeleger et al., 1998). Significant loss of viable bacteria occurs when the bacteria are left at room temperature and atmosphere for only $10 \mathrm{~min}$. Therefore, when working with $C$. jejuni, the amount of time the bacteria are out of incubators and microaerophilic atmosphere must be limited.

\section{Media}

Many types of media have been used to culture $C$. jejuni. Mueller-Hinton medium, blood agar, Columbia blood agar, and BBL medium are commonly used. However, Mueller Hinton ( $\mathrm{MH})$ has the highest recovery rate and is recommended in this unit ( $\mathrm{Ng}$ et al., 1985). Campylobacter defined medium was developed by Leach and colleagues (Leach, 1997). Although a minimal medium has not been developed that supports C. jejuni growth, the defined medium can be used to adjust components based on experimental needs.

\section{Growth Conditions}

C. jejuni can grow in both static and shaking broth cultures. The stringent growth conditions do not lend themselves well to a shaking apparatus. Some tri-gas incubators are equipped to have an internal shaker; however, most are not, and the gas packs in bags or jars do not always allow shaking conditions. $C$. jejuni grown in shaking cultures grow faster than those in static culture. Aggregates form when $C$. jejuni is grown in static broth (Joshua et al., 2006). This aggregation phenotype has been studied extensively, and several genes involved in this have been identified. Based on availability, the protocols suggested in this unit use static growth as the standard.

\section{Strain Selection}

Several isolates of $C$. jejuni have been used in previous research (Table 8A.1.1). Strain selection is important, and depends upon the proposed research. Strains have been isolated from chicken, human, and environmental sources. Phenotypic variation among strains from varying sources has been observed for many traits including invasion, cytolethal distending toxin (cdt) production, chick colonization, lipooligosaccharide (LOS)

Table 8A.1.1 Most Commonly Used C. jejuni Strains ${ }^{a}$

\begin{tabular}{lccl}
\hline & Sources & Sequences & Notes \\
\hline 260.94 & Human & N & GBS $^{b}$-associated; O:41 serotype \\
480 & Human & N & Electrocompetent; highly invasive \\
811681116 & Human & N & Lab strain \\
11168 & Human & Y & Gaynor (2004) \\
$81-176$ & Human & Y & Contains pVir, highly virulent \\
& & & (Bacon, 2000) \\
BTI & Chicken & N & - \\
CG8245 & Human & N & - \\
F38011 & Human & N & - \\
M1 & Environment & N & - \\
RM1221 & Human & Y & - \\
\hline
\end{tabular}

Laboratory Maintenance of C. jejuni

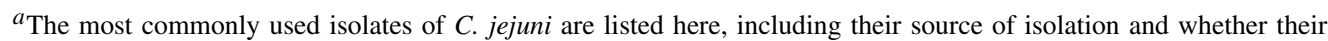
genome has been sequenced to date. Strains can be acquired from individual labs working on the particular isolate. ${ }^{b}$ GBS, Guillain-Barré Syndrome.

\section{A.1.2}




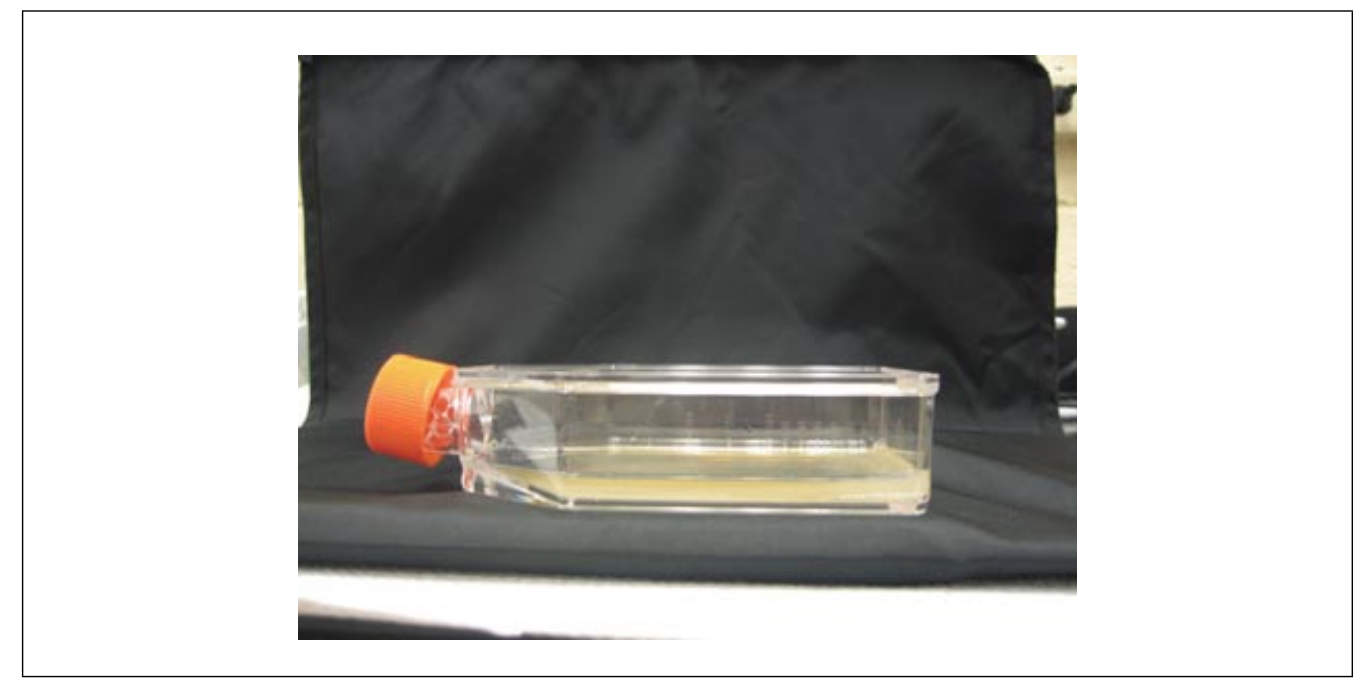

Figure 8A.1.1 Preparation of biphasic medium. In a $75-\mathrm{cm}^{2}$ tissue culture flask, $20 \mathrm{ml}$ of $\mathrm{MH}$ agar were poured and solidified, followed by $20 \mathrm{ml}$ of $\mathrm{MH}$ broth.

production, and natural transformation. The ability of $C$. jejuni to undergo natural transformation has led to a high degree of horizontal gene transfer between $C$. jejuni strains, leading to high genetic diversity among $C$. jejuni strains. $C$. jejuni strains 81-176 and 11168 are well characterized strains, and the most commonly used in pathogenesis studies.

\section{Growth Curves}

Mueller Hinton agar is the recommended medium for standard growth curves of $C$. jejuni. Biphasic MH medium, prepared in tissue culture flasks (Fig. 8A.1.1), is the classic way to perform growth curves. The biphasic medium is made using 50\% $\mathrm{MH}$ agar and $50 \%$ MH (poured after the agar solidifies). Note that MH agar should never be microwaved for $C$. jejuni agar plates and biphasic media.

Biphasic medium is recommended for growing conditions outside of a tri-gas incubator, such as in bags or sealed containers. If a tri-gas incubator is used, growth curves can be performed in $\mathrm{MH}$ broth alone. However, no volume below $1 \mathrm{ml}$ should be used, due to poor recovery and inconsistent growth.

Each time point should be an individual sample. Static growth of $C$. jejuni results in aggregative bacterial raft formation, and disruption of this growth has led to variable and inconsistent growth. Therefore, each time-point should be separate and undisturbed. Furthermore, each time-point should be done in triplicate to obtain a statistically significant average. Growth curves are normally performed for 36 or $48 \mathrm{hr}$.

The inoculum for a growth curve is $1 \times 10^{6} \mathrm{cfu} / \mathrm{ml}$. From 16- to 18 -hr plates, C. jejuni should be resuspended to an $\mathrm{OD}_{600}$ of 0.4 . Dilute this suspension 1:10. Inoculate $80 \mu \mathrm{l}$ of this dilution into $20 \mathrm{ml}$ of medium. A standard growth curve, measured in cfu/ml over $48 \mathrm{hr}$, is shown in Figure 8A.1.2.

CAUTION: Campylobacter jejuni is a Biosafety Level 2 (BSL-2) pathogen. Follow appropriate guidelines and regulations for the handling of pathogenic microorganisms. Proper hand washing is essential, as $C$. jejuni has been shown to cause gastroenteritis at a small dose (10 to 100 organisms). See UNIT 1A.l and other pertinent resources for more information. 


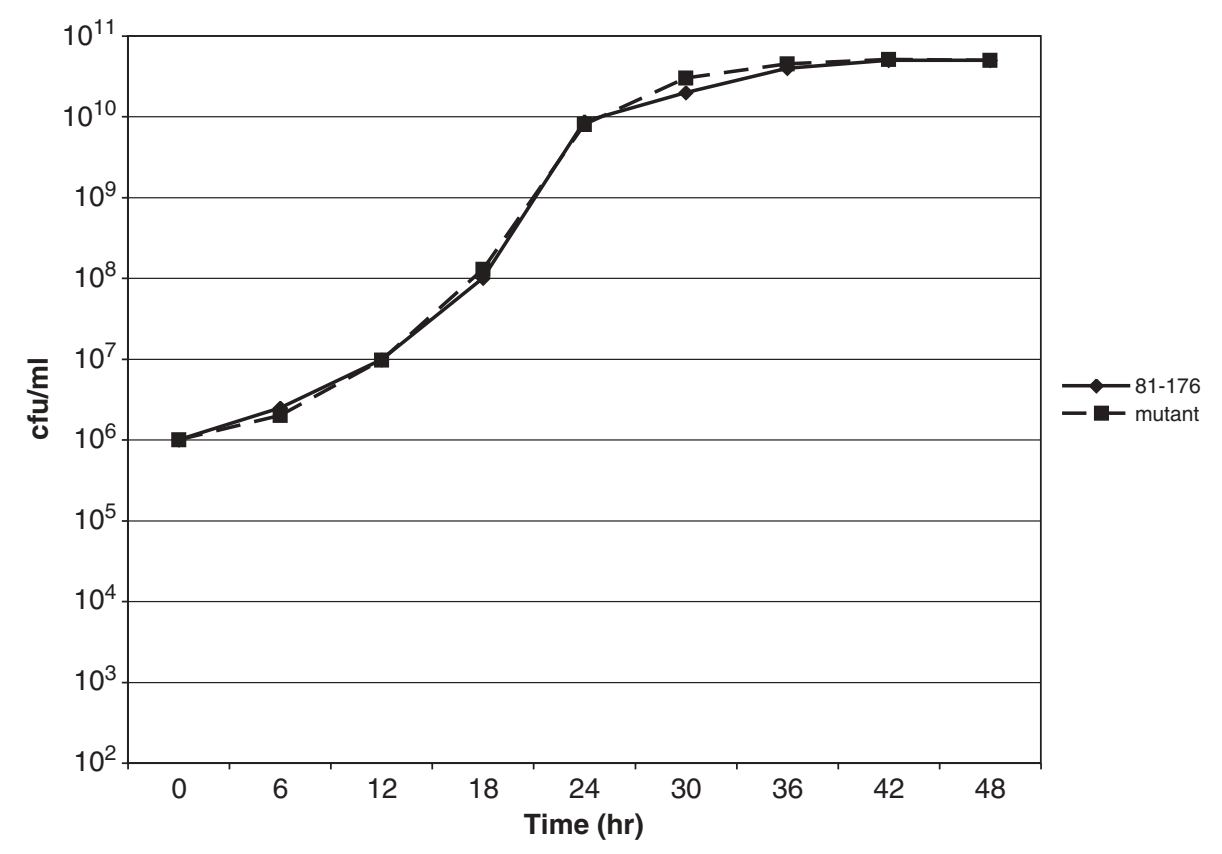

Figure 8A.1.2 Growth curve in biphasic medium of two different isolates of $C$. jejuni 81-176.

BASIC PROTOCOL 1

Laboratory Maintenance of C. jejuni

8A.1.4

\section{GROWTH OF C. JEJUNI FROM A FROZEN STOCK}

C. jejuni is ill equipped for adjusting to changing environments in the lab, especially after being taken out of the freezer. The protocol below supports the best usable $C$. jejuni growth from frozen stocks. Time for growth depends on atmospheric conditions. The protocol outlined below uses Mueller Hinton (MH) broth or MH agar containing $10 \mu \mathrm{g} / \mathrm{ml}$ trimethoprim (TMP) for growing strain 81-176 in a tri-gas incubator. Selective antibiotics can be added to the medium; concentrations commonly used for culturing of and cloning in $C$. jejuni are listed in Table 8A.1.2.

\section{Materials}

C. jejuni frozen stock (Basic Protocol 2)

$100 \times 15-\mathrm{mm}$ Mueller Hinton $(\mathrm{MH})$ agar plates (BD Biosciences, cat. no. 22520; plates are poured in lab) containing $10 \mu \mathrm{g} / \mathrm{ml}$ trimethoprim (antibiotics added in lab)

Mueller Hinton (MH) broth (BD Biosciences, cat. no. 275730) containing $10 \mu \mathrm{g} / \mathrm{ml}$ trimethoprim

Equipment for maintaining Campylobacter-specific microaerophilic atmosphere (see Strategic Planning)

Additional reagents and equipment for streaking bacteria (APPENDIX 4A)

1. From frozen stock (in MH broth plus $20 \%$ glycerol; see Basic Protocol 2), heavily streak out $C$. jejuni strain on $100 \times 15-\mathrm{mm}$ Mueller Hinton agar plates containing $10 \mu \mathrm{g} / \mathrm{ml}$ trimethoprim. Grow for 16 to $20 \mathrm{hr}$ in microaerophilic conditions (see Strategic Planning).

Growth in a tri-gas incubator allows more rapid growth, while bag plating may require up to $48 \mathrm{hr}$ of growth. See Strategic Planning for additional discussion of atmospheric conditions required for growing $C$. jejuni.

Streaking of bacteria on agar plates is described in APPENDIX 4 A. 
Table 8A.1.2 Concentrations of Antibiotics Used in C. jejuni Research

\begin{tabular}{ll}
\hline Antibiotic & Concentration \\
\hline Kanamycin & $50 \mu \mathrm{g} / \mathrm{ml}$ \\
Chloramphenicol & $15-20 \mu \mathrm{g} / \mathrm{ml}$ \\
Streptomycin & $100 \mu \mathrm{g} / \mathrm{ml}$ to $2 \mathrm{mg} / \mathrm{ml}$ \\
Trimethoprim & $10 \mu \mathrm{g} / \mathrm{ml}$ \\
Cefoperazone & $20 \mu \mathrm{g} / \mathrm{ml}$ \\
Nalidixic acid & $30 \mu \mathrm{g} / \mathrm{ml}$ \\
Tetracycline & $12.5 \mu \mathrm{g} / \mathrm{ml}$ \\
\hline
\end{tabular}

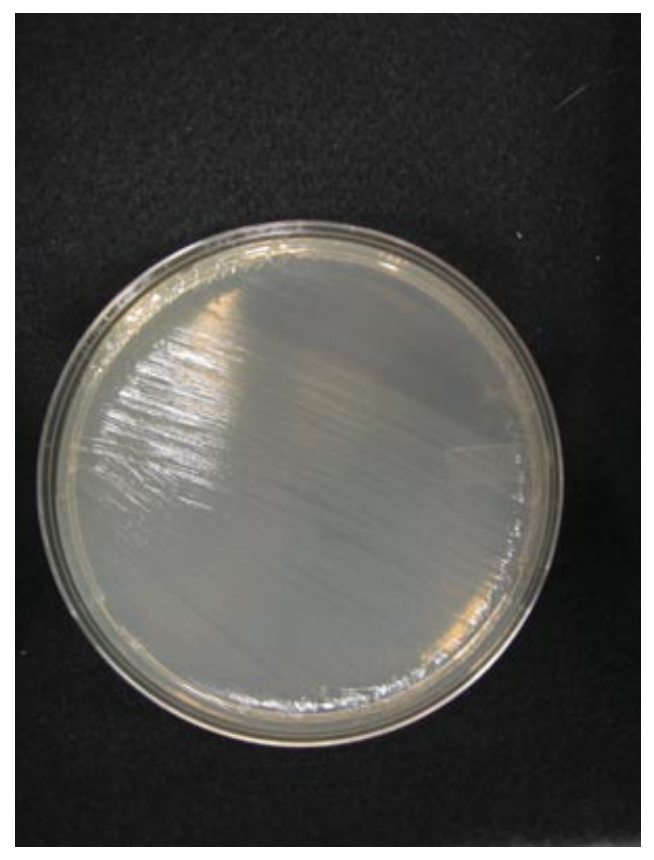

Figure 8A.1.3 Growth of $C$. jejuni 81-176 on an Mueller Hinton agar plate containing $10 \mu \mathrm{g} / \mathrm{ml}$ trimethoprim after $24 \mathrm{hr}$ in a tri-gas incubator.

2. Restreak C. jejuni onto a new Mueller Hinton plate containing $10 \mu \mathrm{g} / \mathrm{ml}$ trimethoprim. Grow for 16 to $20 \mathrm{hr}$ in microaerophilic conditions.

Single colonies will not be observed until culture has been incubated $>24 \mathrm{hr}$. Scrape up a large amount of cells from the lawn (see Figure 8A.1.3).

After this incubation, C. jejuni is ready to be used.

For single colonies, streak for isolated colonies on plates and grow for $48 \mathrm{hr}$ or until individual colonies are observed.

3. Optional: If large amounts are needed, restreak a heavy inoculum onto several Mueller Hinton plates containing $10 \mu \mathrm{g} / \mathrm{ml}$ trimethoprim or inoculate into Mueller Hinton broth containing $10 \mu \mathrm{g} / \mathrm{ml}$ trimethoprim.

4. Optional: Passage $C$. jejuni an additional two to three times.

Further passages from frozen stock are not recommended. 
BASIC

PROTOCOL 2

Stocks of $C$. jejuni should be kept in $20 \%$ glycerol stocks, stored at $-80^{\circ} \mathrm{C}$. Incubations can be performed in a tri-gas incubator or in the other recommended conditions at $37^{\circ} \mathrm{C}$.

\section{Materials}

C. jejuni organisms (cannot be purchased from ATCC or similar sources; must be obtained from individual labs)

$100 \times 15-\mathrm{mm}$ Mueller Hinton $(\mathrm{MH})$ agar plates (BD Biosciences, cat. no. 22520; plates are poured in lab) containing $10 \mu \mathrm{g} / \mathrm{ml}$ trimethoprim (antibiotics added in lab)

Mueller Hinton (MH) broth (BD Biosciences, cat. no. 275730) containing 20\% (v/v) glycerol

Sterile cotton swabs

2-ml cryotubes

1. Heavily streak out $C$. jejuni strain on an MH agar plate containing $10 \mu \mathrm{g} / \mathrm{ml}$ trimethoprim. Grow for 18 to $20 \mathrm{hr}$.

After making a mutant (see UNIT 8A.2), streak for single colonies, which takes 36 to $48 \mathrm{hr}$. Pick a single colony and streak out. Finally, restreak it heavily onto a MH agar plate and carry out the following steps to preserve.

2. Swab bacteria using a sterile cotton swab from plate.

C. jejuni will appear a pink-peach color (Fig. 8A.1.3).

3. Transfer a sufficient quantity of bacteria to $1.5 \mathrm{ml}$ of Mueller Hinton broth containing $20 \%$ (v/v) glycerol in a 2-ml cryotube. Immediately freeze at $-80^{\circ} \mathrm{C}$.

The frozen stock should contain a large amount of bacteria; roughly an $O D_{600}$ of $1.5 \mathrm{or}$ more

4. Never thaw frozen stocks. Instead, scrape off an ice chip from the frozen stock using a sterile implement and plate directly onto $\mathrm{MH}$ agar plates containing $10 \mu \mathrm{g} / \mathrm{ml}$ trimethoprim.

\section{COMMENTARY}

\section{Background Information}

Campylobacter jejuni is the causative agent of campylobacteriosis, a self-limiting gastroenteritis. It is one of the major causes of bacterial-associated gastroenteritis in the United States. C. jejuni has also been linked to Guillain-Barré Syndrome (GBS), an acute autoimmune neuropathy resulting in flaccid paralysis. Research on C. jejuni has been greatly impaired due to poor culturing techniques and genetic tools. C. jejuni was successfully isolated in the early 1970 s, utilizing specialty agar and microaerophilic atmospheric conditions. Originally thought to be primarily a pathogen of animals, $C$. jejuni is now considered one of the main causes of foodborne bacterial gastroenteritis in humans. The original culturing techniques have expanded

Laboratory Maintenance of C. jejuni

8A.1.6 more standardized in recent years. A narrow temperature range, specific media, and specific atmospheric conditions must all be taken into account when growing $C$. jejuni.

\section{Critical Parameters and Troubleshooting}

If no growth is seen from the initial streaking of the frozen stock, or from subsequent re-streaks, the inoculum size or selective antibiotics may be the problem. Try to inoculate the plates with a larger amount of bacteria. A usual amount of frozen stock to be struck out is a chunk of frozen sample the size of a grain of rice. Due to the quantity of passages and relatively short bench life of C. jejuni, it is recommended that two sets of frozen stocks be kept with highly used strains: a backup stock and a frequently used stock. This will help diminish the possibility of contamination. 


\section{Anticipated Results}

A large inoculum of frozen stock of C. jejuni 81-176 should grow after 18 to $24 \mathrm{hr}$. The growth will appear as a hazy lawn. The bacteria will have a pinkish-cream color when scraping the cells from the plate. After re-streaking from the overnight plate, growth of $C$. jejuni 81-176 should be observed after 18 to $20 \mathrm{hr}$. The growth will be hazy. To isolate single colonies, streak for isolation and allow growth for $48 \mathrm{hr}$.

\section{Time Considerations}

Following the protocol, large amounts of viable $C$. jejuni will be available after 36 to 48 $\mathrm{hr}$ in a tri-gas incubator. If gas jars are used, growth will be slower, with usable growth appearing after 40 to $48 \mathrm{hr}$, depending on the amount being streaked.

Freezing a newly constructed $C$. jejuni strain may take 4 to 5 days. Streak from a single colony. Growth will be visible after $48 \mathrm{hr}$. Heavily restreak the growth onto a new $\mathrm{MH}$ agar plate and incubate for another 24 to $36 \mathrm{hr}$.

\section{Literature Cited}

Bacon, D. 2000. Involvement of a plasmid in virulence of Campylobacter jejuni 81-176. Infect. Immun. 68:4384-4390.

Gaynor, E. 2004. The genome-sequenced variant of Campylobacter jejuni NCTC11168 and the original clonal clinical isolate differ markedly in colonization, gene expression, and virulenceassociated phenotypes. J. Bacteriol. 186: 503517.

Hazeleger, W.C. 1998. Physiological activity of Campylobacter jejuni far below the minimal growth temperature. Appl. Environ. Microbiol. 64:3917-3922.

Joshua, G.W., Guthrie-Irons, C., Karlyshev, A.V., and Wren, B.W. 2006. Biofilm formation in Campylobacter jejuni. Microbiology 152:387396.

Leach, S. 1997. Changes with growth rate in the membrane lipid composition of and amino acid utilization by continuous cultures of Campylobacter jejuni. J. Appl. Microbiol. 82: 631640.

$\mathrm{Ng}$, L.-K. 1985. Comparison of basal media for culturing Campylobacter jejuni and Campylobacter coli. J. Clin. Microbiol. 21:226-230.

Young, K., Davis, L.M., and DiRita, V.J. 2007. Campylobacter jejuni: Molecular biology and pathogenesis. Nat. Rev. Microbiol. 5:665-679.
Epsilon

Proteobacteria

8A.1.7 